\title{
Development of overt Cushing's syndrome in patients with adrenal incidentaloma
}

\author{
Luisa Barzon, Francesco Fallo, Nicoletta Sonino and Marco Boscaro ${ }^{1}$ \\ Department of Medical and Surgical Sciences, Division of Endocrinology, University of Padova, Padova and ${ }^{1}$ Department of Internal Medicine, \\ Division of Endocrinology, University of Ancona, Ancona, Italy
}

(Correspondence should be addressed to M Boscaro, Department of Internal Medicine, Division of Endocrinology, University of Ancona, 60100 Torrette, Ancona, Italy; Email clendo@popcsi.unian.it)

\begin{abstract}
Objective: The natural course of adrenal incidentalomas, especially those with subclinical autonomous glucocorticoid production, i.e. subclinical Cushing's syndrome, and the risk that such conditions will evolve towards overt Cushing's syndrome are unknown.

Design: Longitudinal follow-up evaluation of a series of 284 consecutive patients with adrenal incidentaloma.

Methods and results: Out of 284 consecutive patients with adrenal incidentaloma studied at our Institution in the last 15 years, 98 patients ( 23 with subclinical hypercortisolism) underwent surgery. Of 130 non-operated patients with a follow-up of at least 1 year, eight had subclinical hypercortisolism at diagnosis. We describe in detail four patients who developed overt Cushing's syndrome after 1-3 years of follow-up. Only one of these patients had subclinical hypercortisolism at first diagnosis. Estimated cumulative risk for a non-secreting adrenal incidentaloma to develop subclinical hyperfunction was $3.8 \%$ after 1 year and $6.6 \%$ after 5 years. For patients with masses with subclinical autonomous glucocorticoid overproduction, estimated cumulative risk to develop overt Cushing's syndrome was $12.5 \%$ after 1 year.

Conclusions: In patients with adrenal incidentalomas the risk of progression towards overt Cushing's syndrome is not low, at variance with previous reports. A careful biochemical and hormonal followup is advisable in all patients who do not need surgery at first presentation.
\end{abstract}

European Journal of Endocrinology 146 61-66

\section{Introduction}

Adrenal masses discovered by imaging studies performed for unrelated reasons, i.e. adrenal incidentalomas, have become a common clinical problem. In the vast majority of cases these masses are non-hypersecreting adrenocortical adenomas. However, some may show minor endocrine abnormalities with subclinical hyperfunction or represent malignancies (1).

Autonomous glucocorticoid production without specific signs and symptoms of Cushing's syndrome is termed subclinical hypercortisolism or subclinical Cushing's syndrome, a condition found in as many as $5-20 \%$ of adrenal incidentalomas $(1,2)$. The spectrum of subclinical hypercortisolism ranges from mild isolated endocrine abnormalities to atrophy of the contralateral adrenal gland with lasting adrenal insufficiency after unilateral adrenalectomy. Long-term perspective studies assessing the outcome of patients with subclinical hypercortisolism are lacking. The progression towards overt Cushing's syndrome seems, however, to occur only in a very few cases in the short-term (1, $3-9)$.

In the context of our large population of patients with adrenal incidentaloma, we report here in detail four patients who developed overt clinical Cushing's syndrome at follow-up.

\section{Subjects and methods}

During the last 15 years, 284 consecutive patients (170 females and 114 males; mean age $56 \pm 13$ years (S.D.), range $14-77$ years) with adrenal incidentalomas (241 unilateral and 43 bilateral; mean diameter $3.6 \pm 2.5 \mathrm{~cm}$, range $1.0-18 \mathrm{~cm}$ ) were seen at our Institution. Part of the patient population was described in previous studies $(3,10)$. Endocrine evaluation consisted of baseline measurements of plasma cortisol at $0800 \mathrm{~h}$ and 1800 and/or $2400 \mathrm{~h}$, morning adrenocorticotrophin (ACTH), dehydroepiandrosterone sulphate (DHEA-S), 17-hydroxyprogesterone (17-OHP), supine and upright plasma renin activity (PRA) and 


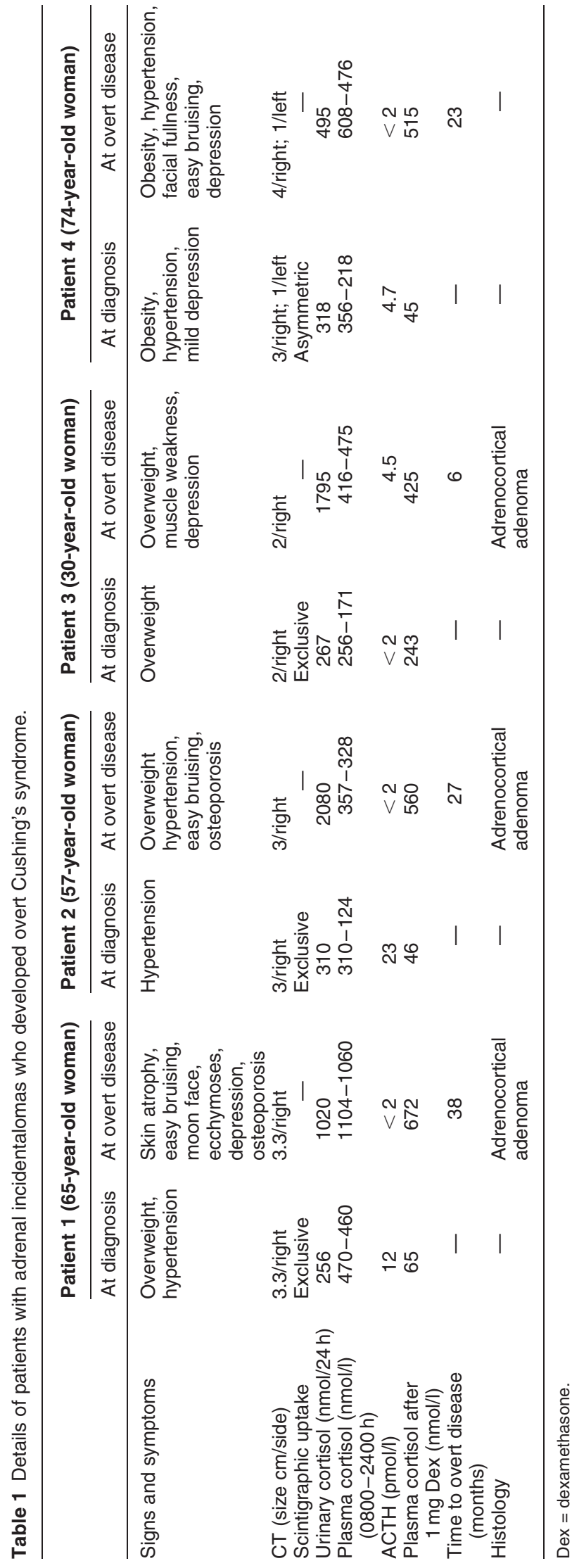

aldosterone, $24 \mathrm{~h}$ urinary cortisol, $24 \mathrm{~h}$ urinary catecholamines and/or metanephrines, as well as dynamic tests (1 mg overnight dexamethasone suppression test, ACTH test, and, in some cases, a corticotrophin-releasing hormone (CRH) test), as described (10). A normal overnight dexamethasone suppression test was defined by a cortisol value $<138 \mathrm{nmol} / \mathrm{l}$ at $0800 \mathrm{~h}$; an exaggerated response to ACTH stimulation was assumed when the ACTH-stimulated 17-OHP levels exceeded $30 \mathrm{nmol} / \mathrm{l}$; adequate glucocorticoid release was defined with a peak cortisol concentration above $550 \mathrm{nmol} / \mathrm{l}$; plasma ACTH and cortisol responses to CRH stimulation were considered normal when their net increases above baseline value, calculated as the mean of the levels recorded at -15 and $0 \mathrm{~min}$, were greater than $4.4 \mathrm{pmol} / \mathrm{l}$ and $200 \mathrm{nmol} / \mathrm{l}$ respectively. Diagnostic morphofunctional work-up included adrenal computed tomography (CT) and/or magnetic resonance imaging (MRI), and ${ }^{75} \mathrm{Se}$-methylnorcholesterol adrenal scintigraphy (10). After initial diagnosis, patients were reinvestigated at 6 and 12 months, and then at yearly intervals by clinical evaluation, routine chemistry, hormone determinations, and morphological assessment (3). Subclinical hypercortisolism was defined as the absence of overt signs and symptoms of hypercortisolism, the presence of cortisol levels not adequately suppressed by overnight $1 \mathrm{mg}$ dexamethasone and at least another abnormal endocrine investigation (plasma cortisol rhythm, urinary cortisol, plasma ACTH). Informed consent was obtained from all subjects, and the investigation was performed in accordance with the principles of the Declaration of Helsinki.

\section{Laboratory methods}

Plasma ACTH was measured by a two-site IRMA (Eurodiagnostic, Amsterdam, The Netherlands; normal range $4-18 \mathrm{pmol} / \mathrm{l}$ ); plasma and urinary cortisol by RIA (Diagnostic Products, Los Angeles, CA, USA; normal range for plasma cortisol at $0800 \mathrm{~h} 138-$ $550 \mathrm{nmol} / \mathrm{l}$ and at $2400 \mathrm{~h}<138 \mathrm{nmol} / \mathrm{l}$; for urinary free cortisol $82-330 \mathrm{nmol} / 24 \mathrm{~h}$ ); PRA, plasma and urinary aldosterone by RIA (Sorin, Saluggia, Italy; normal range for urinary aldosterone 13.8$41.5 \mathrm{nmol} / 24 \mathrm{~h}$; for supine plasma aldosterone $80-$ $280 \mathrm{pmol} / \mathrm{l}$; for supine PRA $1-3 \mathrm{ng} / \mathrm{ml} / \mathrm{h}$ ); plasma 17-OHP by RIA (Diagnostic Systems Labs, Webster, TX, USA; normal range $1.2-10 \mathrm{nmol} / \mathrm{l}$ in males, $0.3-3.6 \mathrm{nmol} / \mathrm{l}$ during follicular phase and $1.2-$ $14.5 \mathrm{nmol} / \mathrm{l}$ during luteal phase in premenopausal females, $0.3-1.8 \mathrm{nmol} / \mathrm{l}$ in postmenopausal females); DHEA-S by RIA (BioRad Labs, Milan, Italy; normal range $0.5-9 \mu \mathrm{mol} / \mathrm{l}$ in males, $1.8-10.5 \mu \mathrm{mol} / \mathrm{l}$ in premenopausal females, $0.3-1.6 \mu \mathrm{mol} / \mathrm{l}$ in postmenopausal females). Urinary free catecholamines were measured by HPLC using an electrochemical detector. The normal range for epinephrine was up to $80 \mathrm{nmol} /$ $24 \mathrm{~h}$, for norepinephrine up to $600 \mathrm{nmol} / 24 \mathrm{~h}$, for 
metanephrine $0.40-1.50 \mathrm{nmol} / 24 \mathrm{~h}$, for normetanephrine $0.6-1.9 \mathrm{nmol} / 24 \mathrm{~h}$. In all these methods intra- and interassay coefficients of variation were $<10 \%$.

\section{Statistical analysis}

Results are expressed as means \pm S.D. Kaplan-Meier survival analysis was used to estimate the likelihood of developing adrenal hyperfunction. All patients entered the life-table when their adrenal mass was first characterized by CT scan or MRI.

\section{Results}

Out of 284 patients, 231 had non-secreting and 53 had secreting tumours (i.e. subclinical hypercortisolism in 32, aldosteronism in six, and adrenal medullary hyperfunction in 15) at initial diagnosis. After the initial evaluation, surgery was performed in 98 patients for subclinical adrenal hyperfunction, or suspicious malignancy, or as the patient's choice. Histological diagnosis was adrenocortical adenoma/hyperplasia in 54, adrenocortical carcinoma in 15, phaeochromocytoma/ganglioneuroma in 16, other pathologies (myelolipoma, cyst, haemorrhage) in 13. At followup, patients with subclinical hypercortisolism who underwent surgery $(n=23)$ showed normalization of the hypothalamic-pituitary-adrenal axis as well as improvement of their hypertension and/or obesity and/or glucose metabolism abnormalities, when present.

Follow-up of at least 1 year (median 56 months, range 1-12 years) was performed in 130 non-operated subjects (3), including eight with subclinical hypercortisolism at diagnosis. Most patients had unchanged CT and/or MRI characteristics of their adrenal mass and did not develop endocrine dysfunction; 16 showed mass enlargement (but no endocrine abnormalities), with appearance of a new mass in the contralateral gland in two; four developed subclinical hypercortisolism and three developed overt Cushing's syndrome without adrenal mass enlargement; three developed hyperfunction (subclinical hypercortisolism in one, overt Cushing's syndrome in one, and catecholamine hypersecretion in one) associated with adrenal mass enlargement. During follow-up no patient developed adrenal malignancies. Estimated cumulative risk for a patient with a non-secreting adrenal incidentaloma to develop either subclinical or overt glucocorticoid hypersecretion was $3.8 \%$ after 1 year and $6.6 \%$ after 5 years. When considering only patients with subclinical autonomous glucocorticoid overproduction, estimated cumulative risk to develop overt Cushing's syndrome was $12.5 \%$ after 1 year.

The four patients (one with subclinical hypercortisolism at first evaluation) who developed overt Cushing's syndrome (Table 1) are described in detail below.

\section{Patient 1}

A 65-year-old woman was referred in 1990 for evaluation of a $3.3 \mathrm{~cm}$ right adrenal mass that was discovered by ultrasonography performed for abdominal pain, and confirmed by CT. The adrenal mass appeared round-shaped, homogeneous, with slight enhancement after i.v. contrast medium. The patient was overweight (body mass index (BMI) $27.5 \mathrm{~kg} / \mathrm{m}^{2}$ ) and had mild hypertension, but no physical signs of hypercortisolism or hyperandrogenism. Laboratory analyses showed normal electrolytes, creatinine, glucose and lipid profile. Endocrine evaluation revealed normal cortisol values but with altered circadian rhythm $(0800 \mathrm{~h}$ $470 \mathrm{nmol} / \mathrm{l}, \quad 2400 \mathrm{~h} 460 \mathrm{nmol} / \mathrm{l}) . \quad{ }^{75} \mathrm{Se}-\mathrm{methylnor-}$ cholesterol scintigraphy showed exclusive uptake at the side of the adrenal mass and non-visualization of the contralateral adrenal gland. At 1 and 2 year follow-up evaluation from diagnosis, mass size was unchanged at $\mathrm{CT}$ and endocrine investigation showed a slight elevation of urinary cortisol values (350$430 \mathrm{nmol} / 24 \mathrm{~h}$ ) with altered circadian rhythm of plasma cortisol.

After 3 years, the patient noticed skin atrophy and easy bruising. Clinical examination revealed moon face, central obesity, proximal muscle weakness, ecchymoses on the legs and back, and depressed mood. Biochemical testing showed hypercholesterolaemia and diabetes mellitus. Urinary cortisol was $1020 \mathrm{nmol} / 24 \mathrm{~h}$, plasma cortisol $1104 \mathrm{nmol} / \mathrm{l}$ at 0800 and $1060 \mathrm{nmol} / \mathrm{l}$ at $2400 \mathrm{~h}$, and not suppressible by $1 \mathrm{mg}$ dexamethasone overnight $(672 \mathrm{nmol} / \mathrm{l})$ nor by $8 \mathrm{mg}$ dexamethasone $(525 \mathrm{nmol} / \mathrm{l})$. Plasma ACTH was not detected and plasma DHEA-S low $(1.2 \mu \mathrm{mol} / \mathrm{l})$. Plasma aldosterone and PRA were within the normal range. Evaluation of haemostatic parameters showed increased prothrombin time (PT) (108\%) and reduced activated partial thromboplastin time (aPTT) (28 s). Adrenal CT was unchanged. Computed bone mineralometry showed marked osteoporosis. The patient underwent open right adrenalectomy, and received perioperative treatment with glucocorticoids, which was continued for about 1 year. Antithrombotic prophylaxis was started immediately after surgery and maintained until normalization of haemostatic parameters. On histological examination, the mass was found to be a benign adrenocortical adenoma. One week after operation, the patient showed normalization of blood pressure and glycaemia. Clinical features of Cushing's syndrome had disappeared and cortisol response to $250 \mu \mathrm{g} \operatorname{ACTH}(1-24)$ i.v. normalized 1 year after surgery. In 1996 and in 1998 adrenal function was retested and found to be normal.

\section{Patient 2}

A 57-year-old woman was admitted in 1990 for evaluation of a $3 \mathrm{~cm}$ right adrenal mass, discovered at 
abdominal ultrasonography performed for abdominal pain. The CT appearance was hypodense, homogenous, with well-defined margins and with rapid enhancement after i.v. contrast medium. The past medical history was negative. On physical examination, the patient had mild hypertension $(160 / 100 \mathrm{mmHg})$, normal weight (BMI $23.9 \mathrm{~kg} / \mathrm{m}^{2}$ ), and a nodular goitre without signs or symptoms of thyroid dysfunction. She also had no physical evidence of hypercortisolism. The laboratory investigation showed normal electrolytes, lipid, and glucose levels. Measurements of urinary catecholamines, plasma aldosterone, PRA, DHEA-S, ACTH, plasma and urinary cortisol, ACTH stimulation test, and $1 \mathrm{mg}$ dexamethasone suppression test were within the normal range. Adrenal scintigraphy showed exclusive ${ }^{75}$ Se-methylnorcholesterol uptake by the adrenal mass. Thyrotrophin was suppressed with normal levels of thyroid hormones and autoantibodies. Thyroid ultrasonography and ${ }^{99 \mathrm{~m}} \mathrm{Tc}$ scintigraphy were consistent with a nodular goitre. Morphological and endocrine data were unchanged at follow-up in 1991.

In 1992 the patient showed clinical features of mild Cushing's syndrome, i.e. weight gain (BMI $26.1 \mathrm{~kg} / \mathrm{m}^{2}$ ), worsening of hypertension, skin atrophy and bruisability. Hormonal study documented not detected ACTH levels, without response to $\mathrm{CRH}$ stimulation, elevated urinary cortisol (1350$2080 \mathrm{nmol} / 24 \mathrm{~h}$ ) and plasma cortisol levels with altered circadian rhythm $(357 \mathrm{nmol} / \mathrm{l}$ at $0800 \mathrm{~h}$ and $328 \mathrm{nmol} / \mathrm{l}$ at $2400 \mathrm{~h}$ ), failure of plasma cortisol to suppress with 1 and $8 \mathrm{mg}$ overnight dexamethasone (560 and $244 \mathrm{nmol} / \mathrm{l}$ respectively). Urinary catecholamines, plasma aldosterone and PRA were normal. Haemostatic parameters showed a hypercoagulable state with decreased aPTT (21 s) and elevated PT (106\%). Adrenal MRI showed a $3 \mathrm{~cm}$ right adrenal mass, hypointense in T1- and T2-weighted images. The patient underwent right open adrenalectomy with removal of a $3.5 \mathrm{~cm}$ benign adrenocortical adenoma. Surgery was performed under hydrocortisone coverage and anticoagulant prophylaxis. After surgery, the patient showed regression of Cushing's features and normalization of blood pressure. During 8 years of follow-up, adrenal function remained normal. In 1997 the patient started treatment with metimazole for subclinical hyperthyroidism.

\section{Patient 3}

A 30-year-old woman was admitted for evaluation of cyclic oedema at the end of 1998. Initial work-up included a pelvic and abdominal ultrasonography, which showed a $1.8 \mathrm{~cm}$ right adrenal mass. Clinical examination showed moderate overweight (BMI $\left.27 \mathrm{~kg} / \mathrm{m}^{2}\right)$, no oedema, normotension $(110 / 70 \mathrm{mmHg})$, no hirsutism or acne, and no other signs of hypercortisolism. Menses were regular. Hormonal evaluation documented normal urinary catecholamines and metanephrines, a slight elevation of plasma aldosterone (470.9 pmol/l supine, $1190 \mathrm{pmol} / \mathrm{l}$ upright) with normal PRA ( $5 \mathrm{ng} / \mathrm{ml}$ per $\mathrm{h}$ supine and $5.6 \mathrm{ng} / \mathrm{ml}$ per $\mathrm{h}$ upright), and normal urinary cortisol $(267 \mathrm{nmol} /$ $24 \mathrm{~h}$ ). Plasma cortisol was normal but had an abnormal circadian rhythm $(256 \mathrm{nmol} / \mathrm{l}$ at $0800 \mathrm{~h}$ and $171 \mathrm{nmol} /$ $\mathrm{l}$ at $2400 \mathrm{~h}$ ) and was not suppressed by $1 \mathrm{mg}$ overnight dexamethasone $(243 \mathrm{nmol} / \mathrm{l})$. ACTH levels were below $2 \mathrm{pmol} / \mathrm{l}$. Responses of cortisol and 17-OH-P to $250 \mu \mathrm{g}$ $\operatorname{ACTH}(1-24)$ i.v. were normal. Abdominal CT scan confirmed the presence of a $2 \mathrm{~cm}$ round-shaped, welldefined, solid, hypodense right adrenal mass. Adrenocortical scintiscan showed exclusive ${ }^{75}$ Se-methylnorcholesterol uptake by the right adrenal mass.

Six months later, she presented with the first symptoms of hypercortisolism, including asthenia and depressed mood. Glucose and lipid profiles were within the normal range. Urinary cortisol was elevated (1795 and $1407 \mathrm{nmol} / 24 \mathrm{~h}$ ). Plasma cortisol showed no circadian rhythmicity $(416 \mathrm{nmol} / \mathrm{l}$ at $0800 \mathrm{~h}$ and $475 \mathrm{nmol} / \mathrm{l}$ at $2400 \mathrm{~h}$ ), and did not respond to $\mathrm{CRH}$ stimulation nor to 2 and $8 \mathrm{mg}$ dexamethasone suppression (425 and $403 \mathrm{nmol} / \mathrm{l}$ respectively). Plasma ACTH was low $(4.5 \mathrm{pmol} / \mathrm{l}$ ) and unresponsive to $\mathrm{CRH}$ (peak $5.7 \mathrm{pmol} / \mathrm{l})$. Plasma aldosterone and PRA values were normal, and DHEA-S was low $(0.52 \mu \mathrm{mol} / \mathrm{l})$. Evaluation of haemostatic parameters showed increased PT $(>110 \%)$ and reduced aPTT (27 s). At adrenal CT the right adrenal mass was unchanged. The patient underwent open right adrenalectomy and received peri- and postoperative glucocorticoid replacement therapy and anticoagulant prophylaxis. On histological examination, the mass was found to be a benign adrenocortical adenoma of $3 \mathrm{~cm}$ in diameter. At 1 year follow-up, the hypothalamic-pituitary-adrenal axis function had recovered. The patient lost weight and was helped for her residual mood disturbance by a short cognitive-behavioural psychotherapy.

\section{Patient 4}

A 74-year-old woman was admitted in 1997 for evaluation of a $3 \mathrm{~cm}$ right adrenal mass discovered at abdominal ultrasonography performed for dyspepsia. The past medical history included migraine and mild depression for about 30 years, and a 3 year history of hypertension $(170 / 100 \mathrm{mmHg})$. On physical examination, the patient had truncal obesity (BMI $32 \mathrm{~kg} / \mathrm{m}^{2}$ ). Blood chemistry profile was normal, including glycaemia and lipids. Hormone evaluation showed low DHEA-S $(0.26 \mu \mathrm{mol} / \mathrm{l})$, urinary cortisol at the upper limit of normal $(318 \mathrm{nmol} / 24 \mathrm{~h})$, and normal suppressibility of plasma cortisol after $1 \mathrm{mg}$ dexamethasone overnight. Cortisol and 17-OH-P responses to ACTH stimulation were normal. Adrenal MRI showed a $3 \mathrm{~cm}$ right adrenal mass and a $1 \mathrm{~cm}$ left adrenal mass, with radiological features of benignity. ${ }^{75}$ Se-methylnorcholesterol scintigraphy showed prevalent uptake by the right mass 
and visualization of the contralateral adrenal gland. After 1 year, at MRI the appearance of the two adrenal masses was unchanged. Endocrine evaluation revealed not detected plasma ACTH, and increased urinary cortisol $(447 \mathrm{nmol} / 24 \mathrm{~h})$. Plasma cortisol lowered to $141 \mathrm{nmol} / \mathrm{l}$ after $1 \mathrm{mg}$ dexamethasone administration. The patient was unavailable for further controls for another year, and then presented with a $5 \mathrm{~kg}$ weight gain, facial fullness, skin bruisability, and worsened depression. ACTH was not detected, urinary cortisol was $495 \mathrm{nmol} / \mathrm{l}$, plasma cortisol was slightly elevated without circadian rhythm $(608 \mathrm{nmol} / \mathrm{l}$ at $0800 \mathrm{~h}$ and $476 \mathrm{nmol} / \mathrm{l}$ at $2400 \mathrm{~h}$ ), and was not suppressed after 1 and $8 \mathrm{mg}$ dexamethasone $(515$ and $427 \mathrm{nmol} / \mathrm{l}$ respectively). ACTH and cortisol did not respond to $100 \mu \mathrm{g}$ ovine CRH i.v. A hypercoagulable state was documented, i.e. increased PT $(109 \%)$ and reduced aPTT (28.8 s). Adrenal CT documented an increase of the right adrenal mass diameter to $4 \mathrm{~cm}$, whereas the left adrenal mass was unchanged. Since the patient refused surgery, she was treated with the steroid synthesis inhibitor aminoglutethimide at a dose of $500 \mathrm{mg} /$ day, which was sufficient to keep urinary cortisol levels within the normal range. An improvement of clinical features and mood disturbances occurred thereafter.

\section{Discussion}

The prevalence of Cushing's syndrome is low and has been estimated to be six to eight cases per million, with ACTH-independent forms (i.e. adrenal adenomas and carcinomas) accounting for about $15-20 \%$ of cases (11). At variance, the prevalence of subclinical Cushing's syndrome seems to be much higher, representing $5-20 \%$ of patients with adrenal incidentalomas $(1,2)$. Thus, given the relatively high prevalence of adrenal incidentalomas (up to $5 \%$ in CT series), and the relatively low prevalence of ACTH-independent Cushing's syndrome, one could assume that the progression towards the overt condition is not very common. However, long-term follow-up studies in large series are lacking, and data in the literature on progression from adrenal incidentaloma to autonomous glucocorticoid oversecretion are scanty (3-5, $9)$. At variance with previous studies $(6-8)$, the risk for an adrenal incidentaloma to evolve towards overt Cushing's syndrome was not very low in our large patient population, suggesting that such an assumption should be reconsidered.

Of the four patients who developed Cushing's syndrome reported here, only patient 3 already had subclinical hypercortisolism at the time of adrenal mass discovery. Patients 1,2 and 4 presented with normal hypothalamic-pituitary-adrenal function, although they had a risk to develop adrenal hyperfunction based on the adrenal mass size and scintigraphic pattern, as shown previously by analysis of risk factors (3). Their management was the same as for other patients with adrenal forms of the disease, i.e. adrenalectomy with postoperative anticoagulant prophylaxis (because of the increased thromboembolic risk) and glucocorticoid substitution in the following months, waiting for the hypothalamic-pituitary-adrenal function to recover. When surgery was not performed, control of hypercortisolism was obtained by pharmacological treatment (12). Development of glucocorticoid hypersecretion may be associated with a slight increase of mass size, as in patient 4 , but generally this is not a sign of malignancy (3).

Our results indicate that in patients with adrenal incidentalomas the risk of progression towards overt Cushing's syndrome is not low. Therefore, a careful biochemical and hormonal follow-up is advisable in patients who do not need surgery at first presentation. Moreover, subclinical hypercortisolism itself may carry an increased risk for steroid-induced negative effects (i.e. hypertension, metabolic disturbances). As in previous observations $(10,13-16)$, an amelioration of isolated clinical or biochemical abnormalities was obtained after surgery in our patients with this condition. The potential benefit of adrenalectomy in these patients should always be considered.

\section{References}

1 Barzon L \& Boscaro M. Diagnosis and management of adrenal incidentalomas. Journal of Urology 2000163 398-407.

2 Reinke M. Subclinical Cushing's syndrome. Endocrinology and Metabolism Clinics of North America 200029 43-56.

3 Barzon L, Scaroni C, Sonino N, Fallo F, Paoletta A \& Boscaro M. Risk factors and long-term follow-up of adrenal incidentalomas. Journal of Clinical Endocrinology and Metabolism $1999 \mathbf{8 4}$ $520-526$.

4 Hensen J, Buhl M, Bahr V \& Oelkers W. Endocrine activity of the 'silent' adrenocortical adenoma is uncovered by response to corticotropin-releasing hormone. Klinische Wochenschrift 1990 $68608-614$.

5 Jockenhovel F, Kuck W, Hauffa B, Reinhardt W, Benker G, Lederbogen $\mathrm{S}$ et al. Conservative and surgical management of incidentally discovered adrenal tumors (incidentalomas). Journal of Endocrinological Investigation 199215 331-337.

6 Barry MK, van Heerden JA, Farley DR, Grant CS, Thompson GB \& Ilstrup DM. Can adrenal incidentalomas be safely observed? World Journal of Surgery 199822 599-603.

7 Terzolo M, Osella G, Alì A, Borretta G, Cesario F, Paccotti P et al. Subclinical Cushing's syndrome in adrenal incidentaloma. Clinical Endocrinology $1998 \mathbf{4 8} 89-97$.

8 Siren J, Tervahartiala P, Sivula A \& Haapiainen R. Natural course of adrenal incidentalomas: seven-year follow-up study. World Journal of Surgery 200024 579-582.

9 Mantero F \& Arnaldi G. Management approaches to adrenal incidentalomas: a view from Ancona, Italy. Endocrinology and Metabolism Clinics of North America 200029 107-111.

10 Barzon L, Scaroni C, Sonino N, Fallo F, Gregianin M, Macrì C et al. Incidentally discovered adrenal tumors: endocrine and scintigraphic correlates. Journal of Clinical Endocrinology and Metabolism $19988355-62$. 
11 Ross NS. Epidemiology of Cushing's syndrome and subclinical disease. Endocrinology and Metabolism Clinics of North America $199423539-546$.

12 Boscaro M, Barzon L, Fallo F \& Sonino N. Cushing's syndrome. Lancet $2001357783-791$.

13 Reincke M, Nieke J, Krestin GP, Saeger W, Allolio B \& Wienkelmann W. Preclinical Cushing's syndrome in adrenal 'incidentalomas': comparison with adrenal Cushing's syndrome. Journal of Clinical Endocrinology and Metabolism $1992 \mathbf{7 5}$ 826-832.

14 Ambrosi B, Peverelli S, Passini E, Re T, Ferrario R, Colombo P et al Abnormalities of endocrine function in patients with clinically 'silent' adrenal masses. European Journal of Endocrinology 1995 $132422-428$.
15 Rossi R, Tauchmanova L, Luciano A, Di Martino M, Battista C, Del Vescovo L et al. Subclinical Cushing's syndrome in patients with adrenal incidentaloma: clinical and biochemical features. Journal of Clinical Endocrinology and Metabolism $2000 \mathbf{8 5}$ $1440-1448$.

16 Marioka M, Fujii T, Matsuki T, Jo Y, Kobayashi T, Tanaka H et al. Preclinical Cushing's syndrome: report on seven cases and review of the literature. International Journal of Urology 20007 126-132.

Received 25 July 2001

Accepted 26 September 2001 